\title{
Percutaneous reimplantation of a pulmonary valved stent in sheep: A potential treatment for bioprosthetic valve degeneration
}

\author{
Yuan Bai, MD, ${ }^{\text {a }}$ Gang-Jun Zong, MD, ${ }^{\mathrm{c}}$ Hai-Bing Jiang, MD, ${ }^{\mathrm{a}}$ Wei-Ping Li, MD, ${ }^{\mathrm{b}}$ Hong Wu, MD, ${ }^{\mathrm{a}}$ \\ Xian-Xian Zhao, MD, and Yong-Wen Qin, MD ${ }^{\mathrm{a}}$
}

\begin{abstract}
Objective: Percutaneous pulmonary valve replacement has been recently introduced into clinical practice. Patients with transcatheter pulmonary valve replacement will definitely face the problems of valve degeneration. In addition to surgical re-replacement of the degenerated bioprosthetic valves, we studied the replacement of degenerated bioprosthetic valves with transcatheter reimplantation of stent-mounted pulmonary valves.
\end{abstract}

\begin{abstract}
Methods: Percutaneous pulmonary valve replacement was first performed in 6 sheep used a homemade valved stent. Two months after the initial procedure, the 6 sheep previously implanted with a valved stent underwent the same implantation procedure of a pulmonary valved stent. Hemodynamic assessment of the bioprosthetic pulmonary valve was obtained by echocardiography immediately post-implant and at 2 months follow-up.

Results: All 6 sheep had successful transcatheter stent-mounted pulmonary valve replacement in the first experiment. After 2 months, reimplantation was successful in 5 sheep but failed in 1 sheep because the first valved stent was pushed to the bifurcation of the pulmonary artery by the delivery sheath. Echocardiography confirmed the stents were in the desired position during the follow-up. The remaining 5 sheep with normal valvular and cardiac functionality survived for 3 months after implantation.
\end{abstract}

Conclusion: Transcatheter stent-mounted bioprosthetic pulmonary valve reimplantation is feasible in an animal model and more convenient than open chest reimplantation.

Since the first case of transcatheter pulmonary valve replacement was reported in $2000,{ }^{1}$ bioprosthetic valves have become popular for use in clinical transcatheter valve replacement. ${ }^{2,3}$ Bioprosthetic valves used in clinical transcatheter valve replacement are made according to the same standard as the bioprosthetic valves used in surgical valve replacement in terms of valve tissue processing, shaping, and sewing technology. They have good hemodynamic characteristics and do not routinely require long-term anticoagulation. The incidence of thrombosis and other complications related to the valve itself is less than with mechanical valves. Their use in open chest valve replacement is gradually increasing. However, bioprosthetic valves have a higher rate of structural degeneration than homografts, thereby limiting their clinical application. With the development in

\footnotetext{
From the Departments of Cardiology ${ }^{\mathrm{a}}$ and Ultrasonography, ${ }^{\mathrm{b}}$ Changhai Hospital, the Second Military Medical University, Shanghai, China; and Department of Cardiology, ${ }^{\mathrm{c}} 101$ Hospital of PLA, Wuxi, Jiangsu Province, China.

Y. Bai and G.-J. Zong contributed equally to this study.

This study was supported by National High-Tech R and D Program of China (" 863 ", Program) (2006AA02Z4D7).

Disclosures: There is no conflict of interest in this study.

Received for publication Feb 19, 2009; revisions received April 24, 2009; accepted for publication May 18, 2009; available ahead of print June 29, 2009

Address for reprints: Yong-Wen Qin, MD, Department of Cardiology, Changhai Hospital, The Second Military Medical University, Shanghai, 200433, China (E-mail: ywqinsmmu@yahoo.cn).

J Thorac Cardiovasc Surg 2009;138:733-7

$0022-5223 / \$ 36.00$

Copyright (c) 2009 by The American Association for Thoracic Surgery

doi:10.1016/j.jtcvs.2009.05.023
}

valve tissue processing, especially with the dramatic development in research on bioprosthetic valve durability in recent years, 10- and 15-year survivals for patients have increased to the same level as those for mechanical valve replacement. A recent report showed that the 20 -year survival with bovine pericardial valve implantation was more than $90 \%$, which is obviously better than that with bileaflet mechanical valves. ${ }^{4}$ However, there is still debate on the life expectancy of bioprosthetic valves.

Transcatheter valve replacement was only recently introduced into clinical practice. Compared with surgical valve replacement, transcatheter valve replacement is less invasive. When the initial bioprosthetic valves inserted by a catheter have degenerated, how does one deal with the degenerated bioprosthetic valves? To date, a surgical approach is the only option for replacing a degenerated bioprosthetic valve. However, reoperation usually involves more clinical difficulties and higher risk. There are few studies on its application when the initial bioprosthetic valve inserted by a catheter has degenerated. Therefore, we studied the replacement of degenerated bioprosthetic valves with transcatheter reimplantation of stent-mounted pulmonary valves with the aim of providing data for clinical practice.

\section{MATERIALS AND METHODS \\ Percutaneous Stent-Mounted Pulmonary Valve Replacement}

Six healthy and hygienic sheep ( 3 female and 3 male, weighing $26.1 \pm$ $3.7 \mathrm{~kg}$ ) provided by the animal center of the Navy Medicine Research 
Institute (License No. 2002-044) were used in this study. The animals were not fed for 8 hours before the operation. After intramuscular injection of ketamine $(10 \mathrm{mg} / \mathrm{kg})$ and diazepam $(5 \mathrm{mg})$, a venous transfusion access on the foreleg was established. Anesthesia was maintained by intravenous injection of $2.5 \%$ sodium pentobarbital. All procedures were performed in the dorsal recumbent position. The right femoral vein was prepared for catheterization. Electrocardiograms were routinely monitored throughout the whole procedure. The study was approved by the local institutional ethics committee. All handling of the experimental animals conformed to the "Experimental Animal Act of Administration" promulgated by the National Science and Technology Committee in 1988. The stent-mounted valves were implanted in the pulmonary valve position through catheterization via the right femoral vein. The details of the stent and implantation procedure have been reported. ${ }^{5}$ After the procedure, the sheep were returned to the controlled animal facility, where their general health was checked daily. The sheep were fed routinely for 2 months after the experiment and were given an intramuscular injection of penicillin 800,000 units twice daily for 3 days to prevent infection, subcutaneous injection of heparin (2500 IU) twice daily for 3 days, and aspirin $3 \mathrm{mg} / \mathrm{kg}$ orally every day.

\section{Transcatheter Reimplantation of Stent-Mounted Pulmonary Valve}

Two months after the initial procedure, the sheep with valves implanted underwent the same preparation for catheterization and anesthesia. Hemodynamic assessment of the right ventricular outflow tract and assessment of the condition of the bioprosthetic pulmonary valve were obtained by echocardiography through the parasternal long-axis view.

An incision of the left femoral vein was made, and a $7 \mathrm{~F}$ sheath was inserted into the vein. A $6 \mathrm{~F}$ pigtail cardiac catheter was inserted through the sheath. Angiography of the prosthetic pulmonary valve was performed (pressure $800 \mathrm{psi}$, total amount of contrast $20 \mathrm{~mL}$, speed $20 \mathrm{~mL} / \mathrm{s}$ ) to identify the position of the previously implanted stent and to measure the diameter of the prosthetic pulmonary valve. After a guidewire was positioned in the distal section of the left or right pulmonary artery, a $14 \mathrm{~F}$ delivery system was then advanced into the pulmonary artery over the guidewire. The implantation was similar to the previous procedure. Thereafter, the stent was deployed inside the valved stent implanted previously. The delivery system was then removed (Figure 1). The procedure ended with angiographic studies and hemodynamic assessment in the right ventricle and prosthetic pulmonary valve. All animals were given an intramuscular injection with penicillin 800,000 units after the procedure.

\section{Clinical Evaluation of Stent-Mounted Pulmonary Valve Reimplantation}

After reimplanting the stent-mounted pulmonary valve, esophageal echocardiography was performed immediately to assess the function of the prosthetic valve and hemodynamics in the right parasternal long-axis view through the right ventricular outflow tract.

After 2 months, echocardiography was performed again with the same views as before to assess the cardiac function, function of the prosthetic valve, and blood flow velocity in different parts of the stent.
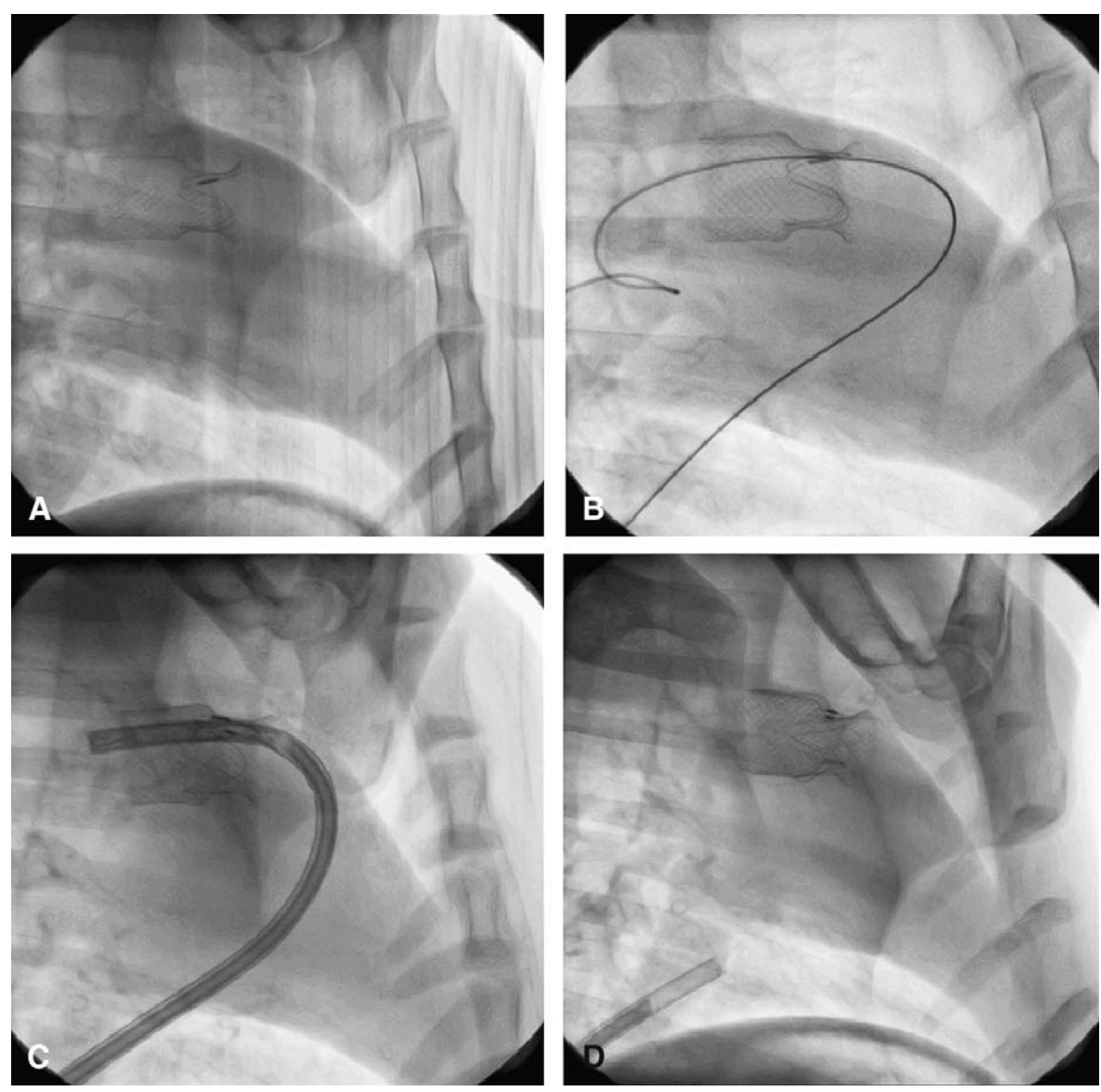

FIGURE 1. Valve stent reimplantation process (representative images). A, The first valved stent in the pulmonary valve annulus. B, The guidewire was deployed across the previous valved stent. C, The second valved stent was delivered to the previously noted location of the pulmonary valve. D, The delivery sheath was retracted, and the second valved stent was released. 


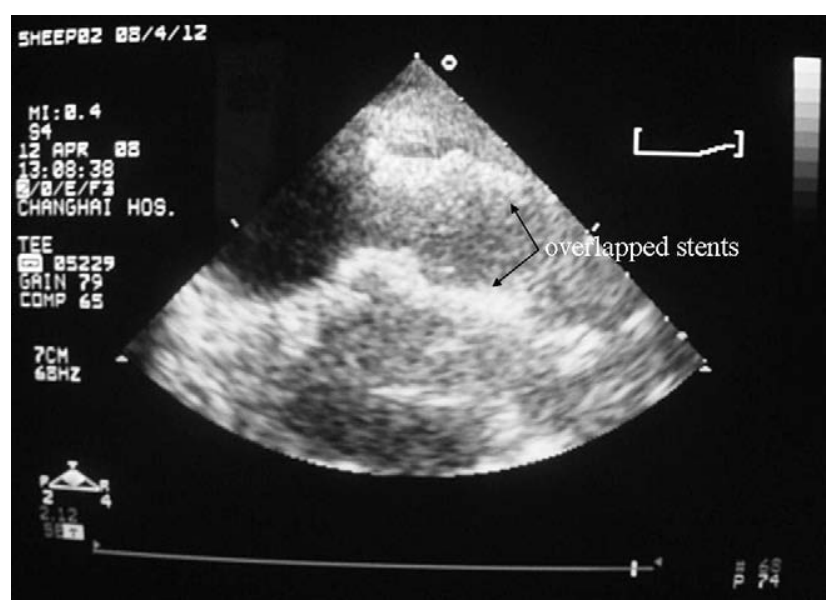

FIGURE 2. Echocardiography shows the 2 valved stents overlapped completely and contacted each other very closely immediately after the reimplantation.

\section{RESULTS}

\section{Results of Implantation}

All 6 sheep had successful transcatheter stent-mounted pulmonary valve replacement in the first experiment. After 2 months, the sheep underwent the second valved stent implantation. Reimplantation was successful in 5 sheep but failed in 1 sheep because the first valved stent was pushed to the bifurcation of the pulmonary artery by the delivery sheath.

The mean pulmonary valve diameter was $14.7 \pm 0.9 \mathrm{~mm}$ as measured by echocardiography before implantation and $15.6 \pm 1.1 \mathrm{~mm}$ as measured by angiography from a body position of 90 degrees left anterior oblique during the implantation. In the first implantation and the reimplantation, the mean diameter at the neck of the valved stent was $19.7 \pm 2.0 \mathrm{~mm}$. The mean duration of the reimplantation was $55.2 \pm 6.8$ minutes. The mean duration of $\mathrm{x}$-ray exposure was $10.5 \pm 2.0$ minutes.

\section{Assessment of the Reimplantation Immediately After the Operation}

Esophageal echocardiography performed immediately after reimplantation showed the following: The position of the prosthetic valve was ideal, the valvular function was normal, there was no regurgitation, and the 2 valved stents were in close contact (Figure 2). On x-ray examination, the 2 valved stents overlapped completely. Angiography in the right ventricle showed that the position of the stents was good. There was no regurgitation involving the prosthetic pulmonary valve (Figure 3).

\section{Assessment Two Months After Reimplantation}

At 2 months after reimplantation, 5 of the experimental sheep were growing well. They ate normally and had gained 4 to $6 \mathrm{~kg}$ of body weight. Echocardiography showed that the

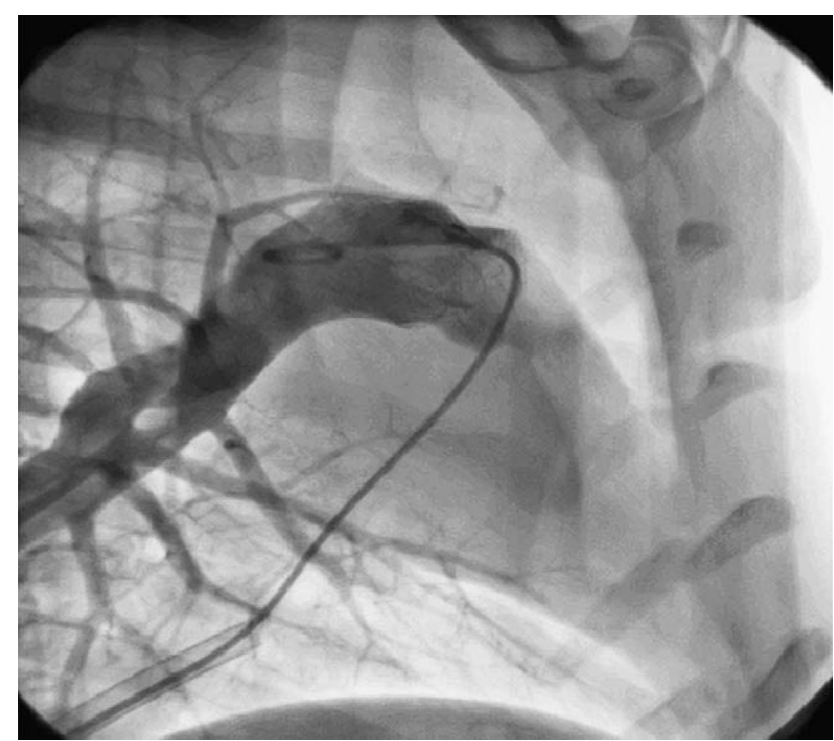

FIGURE 3. Angiography of pulmonary artery. There was no regurgitation involving the 2 prosthetic pulmonary valves immediately after the reimplantation.

valves had normal closure function. Four animals had no regurgitation, and 1 animal had mild regurgitation. The hemodynamics in the valve, over the valve, and in the far section of the stent among all 5 animals that had stentmounted pulmonary valve implantation were similar (Table 1). The ejection fraction was normal $(67.3 \%$ $71.5 \%$ ) (Figure 4).

\section{DISCUSSION}

In the late 1980s, tissue processing for surgical bioprosthetic valves was developed comprehensively. Although the rate of use has increased, many young patients cannot avoid reimplantation of a bioprosthetic valve because of valve degeneration. ${ }^{6,7}$ The development of transcatheter pulmonary valve implantation progressed with accumulation of surgical experience and improvement in catheterization equipment; ${ }^{8}$ however, some problems associated with surgical implantation, such as bioprosthetic valve deterioration

TABLE 1. Peak velocity across the valved stent and heart function assessed by ultrasound 2 months after procedure

\begin{tabular}{lcccc}
\hline $\begin{array}{c}\text { Sheep } \\
\text { no. }\end{array}$ & $\begin{array}{c}\text { Velocity in } \\
\text { valve orifice } \\
(\mathbf{c m} / \mathbf{s})\end{array}$ & $\begin{array}{c}\text { Velocity in } \\
\text { midpiece of } \\
\text { stent }(\mathbf{c m} / \mathbf{s})\end{array}$ & $\begin{array}{c}\text { Velocity in } \\
\text { distal segment } \\
\text { of stent }(\mathbf{c m} / \mathbf{s})\end{array}$ & RVEF (\%) \\
\hline 1 & 226 & 237 & 196 & 70.5 \\
2 & 240 & 243 & 235 & 67.3 \\
3 & 242 & 248 & 245 & 71.2 \\
4 & 230 & 234 & 225 & 68.5 \\
$5^{\text {a }}$ & - & - & - & - \\
6 & 240 & 238 & 236 & 71.5 \\
\hline
\end{tabular}

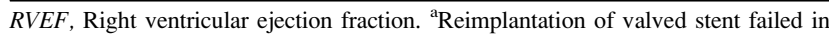
sheep number 5 . 

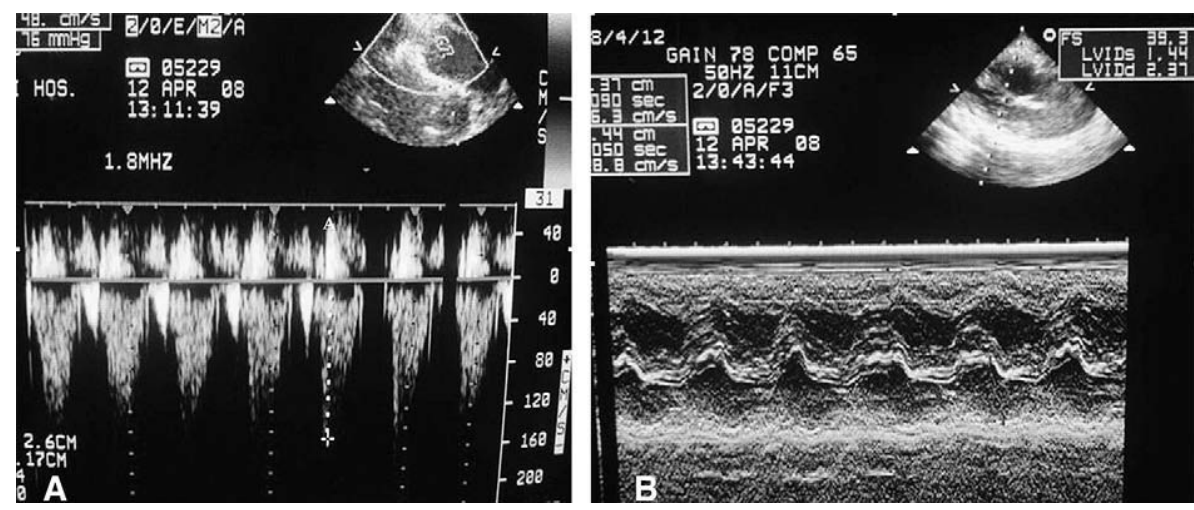

FIGURE 4. Echocardiography evaluation 2 months after reimplantation. A, One sheep had mild regurgitation detected by pulsed-wave Doppler. B, Normal ejection fraction is shown by M-mode echocardiography.

and calcification, also occur in transcatheter valve implantation. ${ }^{9,10}$ In addition to valve deterioration, the metal part of a valved stent can have metal fatigue. If the stent is placed near the right ventricle outflow tract, there may be more extrusion and it may be easily broken. Once a stent is broken, its valvular function will inevitably be affected.

At present, most patients with transcatheter pulmonary valve replacement are relatively young. After a certain number of years, patients will definitely face the problems of valve degeneration and metal fatigue of the stent. Most of these patients have already undergone open chest surgery once or twice, and surgical valve reimplantation incurs a higher risk. ${ }^{11}$

This study used sheep with 2-month transcatheter valve stent implantation as an experimental model to simulate valve degeneration and stent wear, and then reimplantation of a valved stent to achieve the "valve in valve" effect. This study demonstrated 2 problems that remain with the procedure. 1) The position of the stent implanted initially may be affected by the second stent implantation. Therefore, the procedure must be carefully performed. In particular, the sheath holding the second stent must be introduced by guidewire into the initial stent and should not be pushed into the stent directly. In this study, there was 1 case in which the first stent was pushed to the bifurcation of the pulmonary artery by the sheath. On the one hand, this was related to the contact between the sheath and the stent. On the other hand, the first stent had been implanted for only 2 months, and the host endothelial cells and fibrosis tissue barely covered the valved stent, so the stent easily migrated. 2) The second problem concerns how to choose the size of the stent for reimplantation. The diameter of the stent implanted initially was bigger than that of the pulmonary artery so that it could expand the pulmonary artery and fit firmly. If the stent for reimplantation is bigger than the first stent, the pulmonary artery may be expanded excessively. The ideal size of the second stent should be the same as the first stent, which would not only allow the 2 stents to contact each other properly but also avoid excessive expansion of the pulmonary artery. In this study, we used the same sized stent for initial implantation and reimplantation. After follow-up for 2 months, hemodynamics, valvular function, and cardiac function were normal in the reimplanted valved stent. There were no complications, such as valve relocation. Therefore, use of the same sized stent for both implantations is feasible. Our preliminary data also showed that the new type of valved stent was flexible and could be successfully implanted through a catheter 2 or 3 times.

\section{Study Limitations}

Although this study provided some positive experimental results, the study design had certain limitations. The reimplantation was performed too early in that the initially implanted valve was still working normally without wear and tear. These animals have no pulmonary stenosis that would likely be the indication for redo pulmonary valve replacement. With regard to pulmonary stenosis, balloon valvuloplasty may be necessary before valve reimplantation. Therefore, under such circumstances the application of reimplantation to other circumstances is limited. The small sample size in our study is also an obvious limitation. Larger cohorts and longer follow-up durations are needed to provide sufficient reference data for potential clinical application.

\section{CONCLUSIONS}

Transcatheter stent-mounted bioprosthetic pulmonary valve reimplantation is feasible and more convenient than open chest reimplantation. It may become an alternative treatment for young patients with valvular deterioration.

The authors thank the staff of the Department of Cardiology and Catheterization Laboratory of Changhai Hospital and the staff of Shanghai Shape Memory Alloy Co Ltd.

\section{References}

1. Bonhoeffer P, Boudjemline Y, Saliba Z, Merckx J, Aggoun Y, Bonnet D, et al. Percutaneous replacement of pulmonary valve in a right-ventricle to pulmonary-artery prosthetic conduit with valve dysfunction. Lancet. 2000;356:403-5. 
2. Nordmeyer J, Coats L, Bonhoeffer P. Current experience with percutaneous pulmonary valve implantation. Semin Thorac Cardiovasc Surg. 2006;18:22-5.

3. Feldman T, Martin B. Prospects for percutaneous valve therapies. Circulation. 2007; 116:2866-77.

4. Aimoli CG, Nogueira GM, Nascimento LS, Baceti A, Leirner AA, Maizato MJ, et al. Lyophilized bovine pericardium treated with a phenethylamine-diepoxide as an alternative to preventing calcification of cardiovascular bioprosthesis: preliminary calcification results. Artif Organs. 2007;31:278-83.

5. Zong G, Bai Y, Jiang H, Li W, Wu H, Zhao X, et al. Use of a novel valve stent for transcatheter pulmonary valve replacement: an animal study. $J$ Thorac Cardiovasc Surg. 2009;137:1363-9. Epub 2009 Mar 17.

6. Booth C, Korossis SA, Wilcox HE, Watterson KG, Kearney JN, Fisher J, et al. Tissue engineering of cardiac valve prostheses I: development and histological characterization of an acellular porcine scaffold. J Heart Valve Dis. 2002;11: 457-62.
7. Zeltinger J, Landeen LK, Alexander HG, Kidd ID, Sibanda B. Development and characterization of tissue-engineered aortic valves. Tissue Eng. 2001;7:9-22.

8. Cribier A, Eltchaninoff H, Tron C, Bauer F, Agatiello C, Sebagh L, et al. Early experience with percutaneous transcatheter implantation of heart valve prosthesis for the treatment of end-stage inoperable patients with calcific aortic stenosis. $J$ Am Coll Cardiol. 2004;43:698-703.

9. Stark J, Bull C, Stajevic M, Jothi M, Elliott M, de Leval M. Fate of subpulmonary homograft conduits: determinants of late homograft failure. J Thorac Cardiovasc Surg. 1998;115:506-14.

10. Oosterhof T, Meijboom FJ, Vliegen HW, Hazekamp MG, Zwinderman AH, Bouma BJ, et al. Long-term follow-up of homograft function after pulmonary valve replacement in patients with tetralogy of Fallot. Eur Heart J. 2006;27 1478-84.

11. Lutter G, Ardehali R, Cremer J, Bonhoeffer P. Percutaneous valve replacement: current state and future prospects. Ann Thorac Surg. 2004;78:2199-206. 